\title{
CREENCIAS Y PRÁCTICAS DE MADRES GESTANTES DEL BARRIO DE VISTA ALEGRE DE AYACUCHO
}

\author{
BELIEFS AND PRACTICES OF PREGNANT MOTHERS OF THE VISTA \\ ALEGRE NEIGHBORHOOD OF AYACUCHO \\ (D) Cástor Saldaña Sousa ${ }^{*}$ \\ csaldana@udaff.edu.pe \\ ${ }^{1}$ Universidad de Ayacucho Federico Froebel, Ayacucho, Perú
}

*Correspondencia: Cástor Saldaña Sousa. Email: csaldana@udaff.edu.pe

Recibido: 05.11.2020 | Aprobado: 04.12.2020

\section{RESUMEN}

La presente investigación tiene como objetivo describir las creencias y prácticas de la etapa prenatal de madres gestantes que acuden a sus controles prenatales al Centro de Salud de Vista Alegre del distrito de Carmen Alto, Ayacucho, Perú. La metodología empleada es cualitativa y etnográfica, basada en entrevistas abiertas a madres gestantes. Desde el enfoque de la antropología de la salud y desde las teorías del ciclo vital, se concluye que las prácticas y cuidados prenatales por parte de las madres gestantes están influidas por creencias de carácter cultural, religioso y mitológico. Estos factores develan que existe una cultura prenatal local frente a una cultura prenatal occidentalizada, generando dificultades en la comunicación intercultural y, en algunos casos, riesgos para la salud materna y perinatal.

Palabras clave: Gestantes, cultura, antropología de la salud y creencias.

\section{ABSTRACT}

The objective of this research is to describe and interpret the beliefs and practices of the prenatal stage of pregnant mothers who go to their prenatal checkups at the Vista Alegre Health Center in the Carmen Alto district, Ayacucho, Peru. The methodology used is qualitative and ethnographic, based on open interviews with pregnant mothers. From the perspective of health anthropology and life cycle theories, it is concluded that prenatal practices and care by pregnant mothers are influenced by beliefs of a cultural, religious and mythological nature. These factors reveal that there is a local prenatal culture compared to a westernized prenatal culture, generating difficulties in intercultural communication and, in some cases, risks to maternal and perinatal health. Keywords: Pregnant women, culture, anthropology of health and beliefs. 


\section{INTRODUCCIÓN}

El estudio forma parte de un proyecto de investigación titulado: "la etapa prenatal en madres gestantes del Barrio de Vista Alegre del Distrito de Carmen Alto, Ayacucho, Perú", en calidad de docente-investigador de la Universidad de Ayacucho Federico Froebel (UDAFF), Perú. Debido a mi doble perfil como psicólogo y antropólogo, mi interés está en la interdisciplinariedad sobre la base del método etnográfico y mediante el trabajo de campo de primera mano en la convivencia directa y frecuente con los actores sociales y en las instituciones y estructuras culturales en las cuales se reproducen y se llevan a cabo las prácticas cotidianas.

Por otro lado, trato de evitar los reduccionismos disciplinares, en este caso, de la psicología, aportando exclusivamente datos psicométricos, estadísticos o con entrevistas clínicas ligeramente superficiales en cuanto a la captación profunda de la estructura psicodinámica de la personalidad en su contexto sociocultural y biográfico. Desde una perspectiva crítica de la antropología médica y psicoanalítica puede ser una barrera la limitación de tiempo dedicado a cada paciente en el contexto estructural del sistema médico hegemónico.

Este artículo corresponde a una primera etapa de la investigación. En esta primera etapa de la investigación con madres gestantes, el periodo de trabajo de campo se llevó a cabo entre los meses de mayo y noviembre del pasado año dos mil diecinueve. Para este artículo voy a presentar una síntesis etnográfica, teórica e interpretativa de la investigación.

Menciono algunas observaciones previas a modo de barreras y dificultades en el proceso de investigación que se enmarcarían en la antropología médica crítica y la etnografía activa de resolución de problemas como perspectiva teórica y metodología cualitativa. Hay varias situaciones que impiden la fluidez de la investigación en cuanto a las entrevistas con las madres gestantes. Las gestantes acuden a los controles prenatales principalmente por la mañana hasta medio día aproximadamente, variando la afluencia de gestantes al Centro de Salud en los días de la semana. Mis días asignados para el trabajo de campo son sólo jueves y viernes hasta medio día aproximadamente. Otra situación es que cada control prenatal tiene una duración estimada de 45 minutos por gestante con cada obstetra, aunque debido a la afluencia de gestantes muchas veces se reduce a 30 minutos. Esta situación estructural hace que la calidad de atención pueda disminuir. Posteriormente, derivan la madre gestante al consultorio donde me encuentro junto con su historia clínica para la entrevista respectiva. Parte de mi colaboración como psicólogo es administrar una ficha de tamizaje de violencia familiar que el Ministerio de Salud les pide para poder identificar casos de violencia intrafamiliar. Este hecho es una sinergia situacional que puede ser positiva o negativa, o ambas a la vez, dependiendo de la habilidad del etnógrafo para dirigir la investigación hacia los objetivos propuestos. En el aspecto positivo, podemos decir que al ser psicólogo y estar identificado con el uniforme de psicólogo integrado al equipo de salud como un coadyuvante más en la atención a madres gestantes, genera una confianza para la apertura de la gestante en la entrevista clínica y etnográfica accediendo a su universo interno, simbólico, íntimo, psicoemocional y cultural. Como aspecto negativo, no sabemos la percepción de la gestante respecto al imaginario cultural del psicólogo y la limitación del tiempo de atención unido a la estructura de atención sanitaria y administración de la ficha de tamizaje. Esta observación 
se cuestiona desde la antropología médica crítica como una de las objeciones al sistema médico de carácter occidental y a la posibilidad de profundizar en la historia de vida del paciente/actor social.

Otra situación que considerar es que a veces hay ciertas dificultades o barreras en la comunicación entre las madres gestantes y el personal de salud, empezando por las barreras idiomáticas en cuanto al idioma quechua. La mayoría de las madres gestantes son quechuahablantes y la mayoría del personal de salud no son quechuablantes. Esta barrera idiomática hace que las conversaciones no sean fluidas y se limiten a las indicaciones de carácter biológico y fisiológico en cuanto a la prevención de riesgos y la toma de medicamentos, quedando limitada la esfera cultural y afectiva en su exploración.

Por otro lado, puede haber un sesgo de género en cuanto a que el investigador es varón pudiendo generar pudor y desconfianza en las madres gestantes para expresarse afectivamente sobre su proceso de embarazo, sus creencias, representaciones mentales y su vida íntima, sus prácticas respecto al periodo de gestación y los cuidados. Ante este análisis se propone como alternativa futura integrar a asistentes de investigación de género femenino, que sepan hablar quechua, siendo entrenadas para recabar la información necesaria que responda a los objetivos de la investigación, así como elaborar un cuestionario de preguntas abiertas en idioma quechua como guía y referencia de la información requerida, integrando la perspectiva de género como parte del proyecto de investigación.

\section{METODOLOGÍA}

La investigación se caracteriza por la particularidad y la aplicación de una metodología cualitativa etnográfica mediante las entrevistas clínico-terapéuticas y en profundidad, la observación participante y el diario de campo. El propósito es llegar a generar una etnografía sobre la etapa prenatal que integre la antropología médica y la psicología cultural. La población consta de 212 gestantes proyectadas para el año 2019 de las cuales se toma una muestra de 50 gestantes al azar. La población puede variar ligeramente cada año. Cómo técnica cualitativa principal empleamos las entrevistas abiertas en profundidad. Dichas entrevistas recogen información sobre diferentes dimensiones del ser humano: físico, cognitivo, social, cultural, emocional, afectivo, simbólico, mitológico. Las entrevistas se llevaron a cabo durante los controles individuales de las gestantes. En un inicio las entrevistas permiten una primera toma de contacto con las madres gestantes a partir de la derivación de la gestante por el personal obstétrico a la consulta de psicología. Sobre la base de la historia clínica de las gestantes se va estableciendo una conversación informal de carácter psicodinámico y etnográfico en la que se van recabando los datos mensualmente en cada control prenatal.

Los controles prenatales son complementados con visitas domiciliarias por parte de una obstetra a la casa de la gestante para supervisar el proceso de gestación. Esta fase externa del trabajo de campo aún no se ha realizado, excepto una salida que realicé acompañando a la obstetra a la casa de la gestante. Atendiendo a la clasificación de tipos de etnografía que plantea Ángel Aguirre Baztán (2015) adoptamos la etnografía activa de resolución de problemas por ser la más pertinente para nuestro estudio en el campo de la salud: 
De resolución de problemas (se trata de la "etnografía activa por antonomasia", a la que nos referiremos en adelante). Un colectivo tiene una dificultad cultural (cambio, adaptación, cultural, etc.) y recurre al etnógrafo-experto para resolver su problema cultural (pp.172-173).

\section{MARCO TEÓRICO}

Como marco teórico seleccionamos la perspectiva teórica del desarrollo del ciclo de vida de Baltes. Esta perspectiva plantea que el desarrollo en el ser humano se da a lo largo de toda la vida, en múltiples direcciones y dimensiones que interactúan entre sí, con influencias del pasado histórico pudiendo modificar las conductas y la dirección posterior del desarrollo de la persona. Esta teoría, a diferencia de otras corrientes teóricas de la psicología evolutiva, tiene en cuenta la cultura como influyente en el desarrollo de las personas, pudiendo el contexto, cultural e histórico, dirigir a la persona hacia otras direcciones del desarrollo. A diferencia de otras corrientes teóricas, que postulaban que el "desarrollo máximo" culminaba con la adolescencia, la teoría del desarrollo del ciclo de vida defiende la "plasticidad" como concepto aplicado al aprendizaje y al desarrollo de la persona a lo largo de todas las etapas de la vida (Papalia, 2010). Otro enfoque teórico que adoptamos desde las corrientes contextualistas de la psicología del desarrollo es la psicología cultural propuesta por Michael Cole. Cole destaca las siguientes características principales de la psicología cultural:

- Subraya la acción mediada en un contexto.

- Insiste en la importancia del "método genético" entendido ampliamente para incluir los niveles históricos, ontogenético y microgenético de análisis.

- Trata de fundamentar su análisis en acontecimientos de la vida diaria.

- Supone que la mente surge en la actividad mediada conjunta de las personas. La mente es, pues, en un sentido importante, "co-construida" y distribuida.

- Supone que los individuos son agentes activos en su propio desarrollo, pero no actúan en entornos enteramente de su propia elección.

- Rechaza la ciencia explicativa causa-efecto y estímulo-respuesta en favor de una ciencia que haga hincapié en la naturaleza emergente de la mente en actividad y que reconozca un papel central para la interpretación en su marco explicativo.

- $\quad$ Recurre a metodologías de las humanidades, lo mismo que de las ciencias Sociales y biológicas. (Cole, 2003, p.103)

Con este planteamiento queremos conocer como es la etapa prenatal en una muestra de gestantes que acuden a los controles prenatales del Centro de Salud del barrio de Vista Alegre del distrito de Carmen Alto, de la provincia de Huamanga perteneciente al departamento de Ayacucho, Perú. La población de estudio, son migrantes de diferentes comunidades campesinas procedentes de diferentes lugares del departamento de Ayacucho. 
Como objetivo general nos proponemos describir la etapa prenatal de madres gestantes pertenecientes al barrio de Vista Alegre del distrito de Carmen Alto de la ciudad de Ayacucho.

Como objetivos específicos destacamos, por un lado, identificar las concepciones que tienen las gestantes sobre la etapa prenatal y el periodo de gestación y, por otro lado, determinar los cuidados y prácticas que llevan a cabo las gestantes sobre su propio periodo de gestación durante la etapa prenatal.

Teóricamente, no existen estudios de carácter científico a la fecha sobre la etapa prenatal desde el punto de vista etnográfico y desde la antropología médica y la psicología del ciclo vital en el barrio de Vista Alegre del distrito de Carmen Alto, que integre la dimensión cultural, la salud, las creencias y las prácticas.

Por ello, esta investigación es importante porque ayudaría a crear un corpus teórico sobre la etapa prenatal de gestantes pertenecientes al barrio de Vista Alegre, del distrito de Carmen Alto de la ciudad de Huamanga, visibilizando sus características particulares.

Socialmente, es importante porque los resultados son devueltos al Centro de Salud de Vista Alegre para su conocimiento e implementación de programas de intervención comunitaria, sociocultural y psicológica con las gestantes del barrio de Vista Alegre.

Por otro lado, los resultados de la investigación son un aporte para entender la diversidad cultural y psicológica peruana en cuanto a las diferencias comportamentales, idiosincrásicas, creencias, vinculadas a las diferencias regionales y geográficas con relación a la etapa prenatal y su forma de abordarlo social y culturalmente.

En cuanto al estado de la cuestión se realizó una revisión bibliográfica localizando estudios similares a nuestra investigación. A manera de síntesis, mencionamos tres estudios relacionados:

a. Núñez y Herrera (2011) realizaron una investigación sobre los determinantes sociales de la salud relacionados con el embarazo adolescente en el área geográfica de responsabilidad de la unidad de salud "la hachadura" en el municipio de San Francisco Menéndez Departamento de Ahuachapan, en la Universidad del Salvador. Aplicando el método cualitativo con entrevistas en profundidad y la observación directa llegaron a la conclusión de que las adolescentes embarazadas tienen baja escolaridad, en su mayor parte dependen económicamente de sus padres, en su mayoría sufren maltrato por sus familiares, por sus motivos y creencias religiosas no utilizan métodos de planificación familiar, no tienen momentos de esparcimiento adecuado, tienen hábitos higiénicos empobrecidos y la mayoría de las adolescentes viven en hacinamiento.

b. Crispín (1995) investiga las creencias de la mujer embarazada asociadas al cuidado prenatal en la Universidad Autónoma de Nuevo León, México. Aplicando un método cuantitativo que busca determinar cuáles son las creencias de la mujer embarazada que contribuyen al cuidado prenatal. Los resultados generales fueron los siguientes: 
- $\quad$ El 60\% de la muestra tenía una edad menor de 24 años y el $23 \%$ no acudieron al cuidado prenatal; El 54 \% refirió haber cursado estudios inferiores al nivel básico.

- $\quad$ El 20\% no acudió al cuidado prenatal

- $\quad$ El 90\% fueron amas de casa y el 32\% no acudió al cuidado prenatal

- $\quad$ El 80\% refirieron tener pareja y fueron más al cuidado prenatal que las que no tenían pareja.

Crispín llegó a la conclusión de que los cuidados prenatales que refirió realizar la embarazada, están asociados a la susceptibilidad, severidad, beneficios, barreras y señales para la acción, no así el acudir al Control Prenatal. Las creencias percibidas hacia el cuidado prenatal permiten identificar que muchas de ellas percibieron los beneficios en un nivel bajo. La enseñanza del cuidado prenatal a las embarazadas no garantiza su cuidado, pero si se fundamenta en las creencias acerca de los cuidados del embarazo. El apoyo que recibe la embarazada en su entorno familiar es importante en el seguimiento de los cuidados prenatales.

a. Rendon (2012) investiga sobre el significado de las prácticas de cuidado cultural que realizan las gestantes consigo mismas y sus hijos por nacer en el control prenatal en la Universidad Nacional de Colombia. Con un enfoque cualitativo de tipo etnográfico llega a la conclusión de que el significado de las prácticas de cuidado cultural que realizan las gestantes consigo mismas y sus hijos por nacer durante la gestación evidencia como cada cultura determina en las personas lo que sienten, piensan, viven y hacen, en torno a su propio cuidado de la salud, partiendo de los conocimientos y las prácticas que se manejan en cada región donde prevalecen sus propias formas de expresar su cultura y esto se manifiesta en las prácticas de cuidado cultural que se identificaron similares muchas y otras diversas, enmarcadas todas dentro de una estructura social y cultural.

\section{Generalidades sobre la etapa prenatal y el Desarrollo Humano.}

La etapa prenatal la podemos dividir a grandes rasgos en las siguientes subetapas: 1- Concepción 2- Cigoto: desarrollo hasta la segunda semana 3-Embrión: (desarrollo de la $3^{\text {a }}$ hasta la $8^{a}$ semana) 3- Feto (desarrollo de la $9^{a}$ hasta la $38^{a}$ semana). Cada etapa presenta unas características y un desarrollo a nivel fisiológico y cerebral. El desarrollo del ser humano se da desde el mismo momento de la concepción y durante el proceso de embarazo. Este desarrollo no solo es biológico sino también de carácter emocional, cognitivo, psicosocial, cultural y ambiental, factores que incluso pueden pasar desapercibidos y pensar que no influyen ni en el desarrollo prenatal y ni posteriormente en el desarrollo psicosocial de la persona a lo largo de su crecimiento y madurez.

La importancia de la etapa prenatal viene marcada por factores genéticos, factores nutricionales, los lazos afectivos de la pareja, los patrones de comunicación y, las condiciones socioeconómicas del entorno en el marco de un conjunto de patrones culturales y estilos de pensar (Douglas, 1998). El solo hecho de dar a luz es una situación estresante para cualquier mujer que va a ser madre. El proceso que va viviendo la futura 
madre durante el embarazo ha ido generando una serie de cambios a distintos niveles: hormonal, fisiológico, psicológico, social y cultural.

Son muchas las cosas que pueden pasar por la mente de una madre durante el embarazo y sobre todo en los últimos momentos antes de dar a luz y posteriormente en el periodo de puerperio. Emociones de temor y ansiedad, pensamientos de como nacerá el bebé, si va a nacer bien, si va a ser una buena madre, pensamientos de cómo será la nueva situación con la pareja, el impacto emocional de dar a luz en un hospital, el ser atendido por un equipo de médicos y obstetras. Aunque pueda haber patrones comunes en la etapa prenatal, cada persona y cada biografía es única e irrepetible en su forma de vivenciarlo. Además de un conjunto de variaciones como la edad, si se trata de una madre primeriza o si ya ha tenido hijos, la red de apoyo familiar y conyugal.

En la etapa prenatal también se tienen en cuenta los factores teratógenos, es decir, aquellos factores del entorno prenatal que pueden provocar efectos nocivos en el feto, generando con ello diferentes tipos de problemáticas y dificultades en el desarrollo humano, a nivel físico-motor, neurológico, mental, cognitivo y emocional. Algunos ejemplos de factores teratógenos son: el consumo de fármacos, drogas, alcohol por parte de la madre, carencias nutricionales en la madre, problemas mentales previos en los progenitores entre otros factores.

El desarrollo humano no es lineal ni unidireccional, sino multidireccional y multidimensional. Es cierto que el ser humano tiene un desarrollo más marcado desde el nacimiento, la infancia y hasta el inicio de la adolescencia. Los cambios rápidos se pueden observar en la maduración del cerebro y el desarrollo físico hasta la pubertad con los cambios corporales y la aparición de la menarquía en la mujer y las primeras poluciones nocturnas en el varón.

Cada sociedad y grupo humano tiene unos patrones particulares de comportamiento, unos valores culturales, unas costumbres propias que modulan su conducta y la forma de interactuar interpersonalmente, de formar pareja y de entender las relaciones. Es decir, cada cultura y sociedad moldea su propio patrón de desarrollo humano, dentro del margen de variabilidad que le permite el desarrollo biológico y madurativo del sistema nervioso central, el cerebro y la estructura ósea.

Generalización descriptiva de la etapa prenatal de madres gestantes del barrio de Vista Alegre del distrito de Carmen Alto, Ayacucho-Perú

El barrio de Vista Alegre adyacente al Centro de Salud de Vista alegre es un asentamiento humano. Los habitantes proceden de comunidades campesinas aledañas a la ciudad de Ayacucho y del interior del departamento de Ayacucho. En un $70 \%$ aproximadamente, los hijos no son planificados. En cuanto a las costumbres y prácticas tradicionales relacionadas con las madres gestantes, encontramos un grupo de madres gestantes no primerizas que provienen de otros lugares del departamento de Ayacucho que han tenido parto en su domicilio local. En su nueva gestación residiendo en el barrio de Vista Alegre, solicitan al Centro de Salud de Vista Alegre el parto vertical. En este centro de salud no se hace el parto vertical. Las madres gestantes aceptan que sean atendidas bajo el parto horizontal. 
Este cambio de práctica de la cultura prenatal local a la cultura prenatal occidentalizada genera en las gestantes una percepción del cuerpo fuera de su dominio, apareciendo síntomas de estrés, ansiedad y temores ante la nueva situación cultural a la cual tienen que adaptarse, no sólo en el tratamiento del cuerpo biomedicalizado sino en la estructura de creencias de carácter cultural.

En el ámbito educativo, la mayoría de las madres gestantes poseen el nivel de educación primaria y secundaria, siendo pocas las madres gestantes que acceden a la educación superior. Este grupo de personas son asegurados al Seguro Integral de Salud (SIS). La mayoría de las gestantes no tienen una buena situación de pareja, viven con sus padres, dependiendo principalmente de ellos en la parte económica. El machismo sigue vigente; los esposos no quieren acudir al centro de salud y no acompañan a la pareja a los controles prenatales. Les obligan a tener relaciones sexuales y las madres gestantes manifiestan no tener ni sentir placer. Hablar de sexo para las mujeres de 40 años en adelante es un tabú, sienten vergüenza. La mayoría de las gestantes son adolescentes y también hay un grupo de mujeres que son mayores de 40 años. Las mujeres mayores adoptan los métodos anticonceptivos y se aburren de tomarlos porque dicen que les duele la cabeza; al dejar de tomar el anticonceptivo y seguir teniendo relaciones sexuales, es en ese momento donde se quedan embarazadas. Las jóvenes adolescentes sienten vergüenza y tampoco hay una educación sexual, siendo escasa, no adquiriendo responsabilidad respecto a la salud sexual. Las adolescentes piensan que no van a quedar embarazadas. O cuando se embarazan piensan que no van a tener responsabilidades; cuando el niño nace es cuando empiezan a vislumbrar la realidad del nuevo hijo, generando una disonancia cognitiva en su vida. Otro factor es la promiscuidad.

Las gestantes tampoco acuden a todos los controles prenatales, es decir, según relatan el equipo de obstetras, no hay sensibilidad respecto al cuidado de su salud en relación con la atención en el Centro de Salud. Muchas gestantes se afilian al centro de salud mediante el SIS, pero viven en lugares alejados del Centro de Salud y algunas ni acuden. Hay pacientes que no acuden a los controles prenatales aduciendo que no tienen tiempo por motivos de viaje y labores domésticas y de trabajo. Las principales demandas por parte del Centro de Salud y del equipo de obstetras son:

1. La importancia de ser madre y acudir a sus controles prenatales

2. Cumplir con los objetivos y los protocolos del control prenatal

3. Que no tenga una hemoglobina baja y otros parámetros biomédicos y fisiológicos.

Se ha implementado un servicio de psicoprofilaxis a partir de las 26 semanas de gestación en adelante, con la finalidad de que la gestante disminuya sus niveles de ansiedad y estrés en el parto, que el parto sea sin dolor y no sea complicado. Ayuda a las gestantes jóvenes a disminuir temores, ansiedades, estrés, aprendiendo técnicas de relajación y de respiración. Las sesiones se desarrollan una vez a la semana por las tardes. Trabajan con un promedio de 90 gestantes al mes que acuden al control prenatal, haciendo uso de su historia clínica.

A todas las gestantes se les deriva al psicólogo como interconsulta obligatoria. La idea que tienen del psicólogo es que "hay que ir cuando uno está mal de la cabeza". Las 
obstetras le sugieren ir al psicólogo, pero "no van porque dicen que están bien y eso es para cuando estén mal". Cuando se quedan embarazadas dejan de tener relaciones sexuales desde el comienzo porque piensan que la penetración afecta al feto o al niño por nacer. Esta creencia implica que dejan de cuidar la relación conyugal y el afecto entre ambos miembros de la pareja. Los varones piensan que se han equivocado al tener la relación sexual pensando que fue un error involuntario, y muchos terminan teniendo relaciones sexuales con otras mujeres y relaciones extraconyugales. Los varones piensan que su parte intima va a llegar al feto.

Hay pacientes que dan a luz y no se bañan en toda la semana porque piensan que el feto se puede enfermar y que les puede dar aire, una de las enfermedades de filiación cultural propias de la región de los Andes. En el parto, las gestantes prefieren tomar aceite rosado porque piensan que lubrica su parte íntima y tiene el efecto de que el bebé resbala rápido a la hora de dar a luz. Otra práctica es que se frotan en su barriga con el aceite rosado con la idea de provocar una vasodilatación. Otra forma de provocar una dilatación rápida es tomar cerveza negra durante el momento del parto para sea más rápido y fácil. Se hacen arreglar el cuerpo con las comadronas en el embarazo haciéndole suysu, termino quechua que significa "arreglary sobar el vientre para colocar adecuadamente el feto".

En las historias clínicas y mediante el protocolo del control prenatal se ven los riesgos, enfermedades previas, abortos, otros hijos, niveles de hemoglobina, enfermedades de transmisión sexual, grado de instrucción. Estadísticamente hay aproximadamente quince gestantes al mes. Las gestantes que vienen por su control prenatal vienen generalmente por su cuenta, muchas gestantes vienen después de tres meses. El indiciador de salud institucional del Ministerio de Salud (MINSA) refiere que la gestante sea captada tres meses antes de la gestación.

El MINSA sugiere que la gestante tiene que ir a la posta médica para recibir consejería en prevención de discapacidades prenatales tres meses antes de la concepción. Sin embargo, no vienen. Incluso en los tres meses previos al embarazo y tres meses después de concebir deberían tomar ácido fólico que lo brinda el estado gratuitamente. Además de tres meses después para prevenir malformaciones del sistema nervioso central y a nivel neurológico. En los tres meses antes del embarazo, continúa relatándome el equipo de obstetricia, el esposo debería reducir o dejar algún tipo de vicio si lo tuviere, como el alcohol, tener una dieta alimenticia adecuada y, psicológicamente que este estable con la pareja. Se les prohíbe consumir "comida chatarra", incluso se le indica según su periodo menstrual que días son los más fértiles para tener una relación sexual.

Teóricamente, es en el día catorce del ciclo menstrual en donde se presenta la ovulación, viviendo el ovulo entre 48 y 64 horas. Según me relatan el equipo de obstetras, algunos autores diferencian la calidad del bebé, si conciben al principio o al final de la vida del ovulo: "Se dice que si fecunda al comienzo de la vida del ovulo es bueno y si es al final es distinto". Sin embargo, la realidad indica que las madres gestantes suelen acudir al Centro de Salud tres meses después de quedarse embarazada, quizá por sus padres que lo hicieron así anteriormente, quizá por educación, por factores culturales y por qué ocultan el embarazo. Según relatan las informantes, ocultan el embarazo por su condición de adolescente y para no ser criticadas socialmente. 
Una situación particular es que cuando las gestantes acuden al centro de salud antes de los tres meses, el personal de salud le llama la atención, sintiéndose mal las gestantes porque, desde su percepción psicocorporal, sienten que el personal de salud les grita cuando les asisten sobre la situación del embarazo y por esa percepción dejan de venir al centro. El personal de salud generalmente piensa de las gestantes que son descuidadas y no hacen caso a lo que se les indica desde el paradigma obstétrico de la biomedicina. Esto se ha visto en grupos focales según me relata el obstetra integrante del equipo. En cambio, el usuario, en este caso la mujer gestante, piensa que el personal de salud es un sabelotodo, que tiene la verdad, que tiene la razón y no respeta la cultura de ellos.

Por lo tanto, aquí vemos que hay un enfrentamiento de carácter perceptivo-cultural entre dos paradigmas acerca del embarazo: la cultura prenatal local por parte de las gestantes y la cultura prenatal desde la visión obstétrica occidentalizada. El estado civil de las gestantes no es una unión formal de casamiento, sino que es el estado que se denomina cohabitación o pareja de hecho, es decir, la convivencia de la pareja.

Hay bastante incidencia de gestantes adolescentes. Hay incidencia de anemia en gestantes, provocando hemorragias en el momento del parto porque es más propensa y puede morir la madre y, a veces, hasta los dos, la madre y el hijo. Hay un cierto desconocimiento de la gestante sobre la concepción y el proceso de embarazo. No planifican la concepción y, muchas veces, cuando festejan, se embarazan en estado de embriaguez al tomar alcohol.

Muchos casos terminan en aborto.

Destacamos dos datos relevantes del trabajo de campo: Una de las madres gestantes es su segunda gestación. En su primer parto dio a luz en el Hospital Regional de Ayacucho. La gestante relata que durante el parto en la sala del hospital los médicos observaron que el bebé tenía una vuelta y media del cordón umbilical sobre el cuello por lo que decidieron paralizar el proceso de parto durante media hora para ver si el mismo bebé, al moverse en el interior de la barriga, podría desenrollarse el cordón umbilical por sí mismo. En ese lapso, invitan a la madre de la gestante a que pase a la sala de parto para acompañar a su hija.

La gestante relata en la entrevista que su madre le entregó un trozo de tejido que había hilado durante el proceso de gestación y le pidió que la gestante desmenuzara el trozo de tejido hilado en el momento con la creencia e idea de que esa acción y práctica haría que el cordón umbilical se desenrollaría del cuello del bebé. La madre de la gestante salió de la sala con el trozo de tejido deshilachado antes de que volvieran los médicos para continuar con el proceso de parto. Al final, el parto fue exitoso con el nacimiento del bebé sin complicaciones de ningún tipo.

Lo relevante de esta situación es que cuando le pregunté a la gestante que, si pensaba que el nacimiento exitoso del bebé se debía a la decisión médica o a la acción de deshilachar el trozo de tejido, la gestante asume que el desenrolle del cordón umbilical sobre el cuello del bebé se debía a su acción personal de deshilachar el tejido, pensando que había sido su responsabilidad y "culpa" (según relata la gestante) al hilar durante el periodo de embarazo varios meses atrás. Esta experiencia y asunción cognitiva-simbólica 
y cultural la mantiene vigente en el presente periodo de gestación que está atravesando, en el cual no está hilando y tejiendo debido a la creencia que le pueda suceder lo mismo que con el primer hijo.

Hay datos sin transcribir en esta entrevista y en otras entrevistas que reflejan situaciones similares, como, por ejemplo, no escuchar la radio durante el embarazo en ciertos momentos del día y condiciones; no coger la gestante mucho tiempo el gato durante el periodo de embarazo.

El segundo dato relevante se trata de una madre gestante que no está cumpliendo con el protocolo prescrito de tomar el sulfato ferroso y el calcio administrado por el Centro de Salud debido a la creencia transmitida por su madre de que al tomar los medicamentos prescritos por el Centro de Salud el bebé puede engordar.

Para determinar los cuidados y prácticas que las gestantes llevan a cabo durante su periodo de gestación se tienen que complementar las consultas prenatales con las visitas a domicilios en compañía de las enfermeras. La parte preventiva y extramural del Centro de Salud, se hace mediante las visitas domiciliarias para lograr la captación oportuna de la gestante antes de los tres meses de embarazo. A veces por miedo o por vergüenza, acuden a los cuatro, cinco o seis meses de embarazo.

Existe la figura denominada agente comunitario, que es un poblador local que se le ha asignado ese rol y se le ha capacitado y en el cual, confían más las personas gestantes al ser del barrio. Es un mediador cultural entre la comunidad y el personal de salud. A veces el personal de salud tiene una gran brecha de comunicación, debido a que la gran mayoría de pobladores son quechuahablantes y la gran mayoría de los trabajadores de salud no hablan quechua, incluso algunas personas que residen en el barrio y algunas madres gestantes son de la costa.

Hay una entrevista sin trascribir de una madre joven ashaninka, etnia nativa de la selva peruana. Uno de los días del trabajo de campo, la coordinadora del equipo de obstetras me invitó a pasar a la sala de partos para conversar con una joven que recién había dado a luz a su nuevo hijo. El paciente cuando va a al Centro de Salud siente una barrera idiomática con el personal de salud y en los códigos lingüísticos de carácter cultural. Se trata de superar esas dificultades, pero el poblador muchas veces no lo ve de esa perspectiva y entre ellos no se entienden. En este aspecto entra a tallar el agente comunitario, dando las instrucciones y las indicaciones de acuerdo con las directrices del MINSA.

El trabajo del obstetra es preventivo, educar a la mujer en edad fértil, que el embarazo debe ser planificado, debe ser en la edad correcta, de los 18 hasta los 35 años aproximadamente. En la parte preventiva se hacen visitas domiciliarias, se brinda el servicio de planificación familiar. El trabajo obstetra asistencial consiste en brindar el servicio del control prenatal, donde se hacer un seguimiento del proceso evolutivo de la gestante, nutrición, salud sexual reproductiva, se le ensaña que puede tener relaciones sexuales hasta cierta etapa de la gestación, se les enseña signos de alarma: le dicen si le duele mucho la cabeza que vuelva rápido al centro porque puede ser elevación de la 
presión arterial, si tienes escotomas ( ver con chispas) que vaya, si él bebe no se mueve tiene que venir de inmediato, si hay perdida de líquido o sangrado vaginal.

En el servicio de obstetricia ven muchos casos sobre creencias de las gestantes acerca del hilado y otras creencias y tratan de no ir en contra de sus creencias, brindando un servicio integral y brindándole una información clara tratando de aplicar la interculturalidad. Al hacer ecografías, pueden ver si hay algún tipo de riesgo para la madre o para el nuevo ser humano, y se les explica desde el paradigma obstétrico y le dicen que no deben mantear. El manteo es una práctica cultural y local que consiste en que a la gestante la colocan en una manta y dos personas empiezan a mover la manta zarandeando a la gestante. Estos movimientos bruscos en la creencia de la gestante significan que va a hacer que él bebe se acomode en la barriga de la gestante colocándole en la posición correcta en el vientre. La otra parte del proceso del manteo consiste en que el mismo pariente o curandero que buscan simplemente con movimientos o maniobras manuales sobre el vientre de la gestante voltean (le dan la vuelta) al bebe. Las obstetras tratan de trasmitir a las gestantes y educar de que esa práctica cultural es peligrosa porque por algún movimiento brusco se puede dar un desprendimiento de placenta ocasionando la muerte del feto y también pudiendo ocasionar la muerte de la madre; o también enredar más el cordón umbilical o que se ajuste más sobre el cuello del feto.

En muchas ocasiones se ha visto que la gestante no es dueña de su cuerpo y de su gestación, es decir, en su casa, en familia, comenta que la obstetra le ha dicho tal o cual cosa y es ahí donde la familia escucha, la abuelita, el esposo, la suegra, y le dicen: "no, si él bebe está mal te vamos a llevar a tal sitio que él sabe acomodar barrigas". La obstetra expresa en la entrevista que es ahí donde la mujer tiene que decir que la obstetra le ha dicho que no. Le piden que los esposos o algún familiar esté presente para que escuche las orientaciones de la obstetra sobre los cuidados prenatales. Estos casos se manifiestan en gestantes que vienen de zonas rurales.

Los obstetras y las obstetras me relatan que los pobladores mismos de Vista Alegre tienen otra mentalidad porque están más en contacto y con acceso al Centro de Salud. Algunas gestantes tienen miedo de los profesionales cuando se visten con las batas para la atención de parto y también cuando se suben a la camilla. Hay un choque cultural perceptivo en esas experiencias y prácticas en el Centro de Salud que las gestantes pasan por un periodo de asimilación y adaptación.

Viendo estas situaciones y hechos sociales entre dos culturas y percepciones diferentes respecto a la etapa prenatal, el personal de salud está conversando para tratar de implementar una sala de parto adecuándolo a las concepciones, mentalidad, cultura y los pensamientos que las gestantes tienen acerca de su cosmovisión propia sobre el embarazo, parto y puerperio. En este sentido estaríamos planteando un diálogo y una alternativa práctica en la salud intercultural y la antropología médica aplicada.

\section{CONCLUSIONES}

Podemos afirmar que existen un conjunto de creencias, concepciones, representaciones mentales, prácticas y cuidados sobre la etapa prenatal que son de carácter cultural en el conjunto de madres gestantes que acuden al Centro de Salud de Vista Alegre, 
las cuales modulan el comportamiento cultural, social, psicológico y el proceso de gestación en interacción muchas veces limitante y negativa con la atención y los controles prenatales establecidos por la ciencia obstétrica.

Estas actitudes son de naturaleza cultural, que provienen de la cosmovisión y el pensamiento andino, en el que se mezclan rasgos históricos de un pasado precolombino hasta la actualidad manifestándose también el denominado sincretismo religioso. Las dificultades en la comunicación con las gestantes y en el seguimiento de todos los controles prenatales y las indicaciones del personal obstétrico del Centro de Salud de Vista Alegre se debe a que hay dos paradigmas encontrados sobre la etapa prenatal: el paradigma biomédico junto a las directrices del MINSA sobre la etapa prenatal occidentalizada y el paradigma de la etapa y cultura prenatal local, tal como es entendida, concebida y practicada por la población de gestantes que acude al Centro de Salud de Vista Alegre.

\section{REFERENCIAS BIBLIOGRÁFICAS}

Aguirre Baztán, A. (2015). Metodología cualitativa etnográfica. Universidad de Ayacucho Federico Froebel.

Barrientos Núñez, L. A. y Tobar Herrera, V. A. (2011). Determinantes sociales de la salud relacionadas con el embarazo en la adolescencia en el área geográfica de responsabilidad de la unidad de salud la hachadura municipio de San Francisco Menéndez Departamento de Ahuachapan. Enero a junio de 2011. Tesis de maestría en Salud Pública, Universidad del Salvador.

Cole, M. (2003). Psicología cultural. Una disciplina del pasado y del futuro. Ed. Morata.

Douglas, M. (1998). Estilos de pensar: ensayos críticos sobre el buen gusto. Ed. Gedisa.

Jeannette Rendon, Blanca. (2012). Significado de las prácticas de cuidado cultural que realizan las gestantes consigo mismas y sus hijos por nacer en el control prenatal. Tesis de magister en enfermería, Universidad Nacional de Colombia, Colombia.

Papalia, D.E; Wendkos Olds, S.; Duskin Feldman, R. (2010). DESARROLLO HUMANO. Undécima Edición. McGraw-Hill Companies

Quintero Crispín, A. L. (1995). Creencias de la mujer embarazada asociadas al cuidado prenatal. Tesis de maestría en enfermería, Universidad Autónoma de Nuevo León, México.

Saldaña Sousa, C. (2021). Creencias y prácticas de Madres Gestantes del Barrio de Vista Alegre de Ayacucho. Puriq, 3(1), 26-38. https://doi.org/10.37073/puriq.3.1.138 